\title{
A Case of Malignant Melanoma with In-Transit Metastasis That Responded to Intravenous Infusion of Interferon- $\beta$
}

\author{
Masaru Arima ${ }^{a} \quad$ Youhei Iwata $^{a} \quad$ Yusuke Morita $^{a} \quad$ Tsukane Kobayashi $^{\mathrm{a}}$ \\ Ryousuke Sasaki $^{b}$ Kayoko Suzuki ${ }^{b}$ Kayoko Matsunaga ${ }^{a}$ \\ Departments of Dermatology at ${ }^{\mathrm{a}}$ Fujita Health University School of Medicine, Toyoake, \\ and ${ }^{b}$ Kariya Toyota General Hospital, Kariya, Japan
}

\section{Key Words}

Interferon- $\beta \cdot$ Malignant melanoma $\cdot$ In-transit metastasis

\begin{abstract}
A 77-year-old man with a history of surgical resection of malignant melanoma involving the fifth toe of his left foot 14 years ago presented at the Kariya Toyota General Hospital with a 3-month history of skin ulcer at the same site and red nodules on the lower left leg. Malignant melanoma was suspected, and the patient was referred to our department. On examination, a skin ulcer measuring $25 \times 20 \mathrm{~mm}$ was observed at the amputation site on the left foot. In addition, multiple red nodules were observed on the lower left leg. Skin biopsies of the ulcer and nodules revealed recurrent malignant melanoma with in-transit metastasis. Two weeks later, he developed acute myocardial infarction and was hospitalized at the Kariya Toyota General Hospital. One month later, the myocardial infarction ameliorated, and he was transferred to our department. As the myocardial infarction had decreased the patient's tolerance to surgery, interferon- $\beta$ was administered by intravenous infusion. The skin ulcer and red nodules on the lower left leg disappeared 26 weeks after infusion had been initiated. The patient's progress has been satisfactory, with no evidence of recurrence or metastasis at 1 year and 9 months after the initiation of intravenous infusion.

(c) 2014 S. Karger AG, Basel
\end{abstract}


Arima et al.: A Case of Malignant Melanoma with In-Transit Metastasis That Responded to Intravenous Infusion of Interferon- $\beta$

\section{Introduction}

In-transit metastases from malignant melanoma are subcutaneous lymphogenous metastases that invade regional lymph nodes and are commonly observed as a type of recurrence. A clear treatment strategy is yet to be established, and in many instances, physicians struggle while deciding treatment. Here, we report a case of malignant melanoma with in-transit metastasis that responded to intravenous infusion of interferon (IFN)- $\beta$.

\section{Case Presentation}

\section{Patient and Medical History}

The patient was a 77-year-old male with rash on the fifth toe of the left foot and lower left leg. His medical history included hypertension, diabetes, and malignant melanoma that was surgically removed (fifth toe amputation) at our department 14 years ago (fig. 1). Otherwise, his family history was unremarkable. Three months before presentation, a skin ulcer appeared at the same site, and red nodules developed on the lower left leg. Subsequently, the patient consulted the Kariya Toyota General Hospital. Malignant melanoma was suspected, and the patient was referred to our department.

At the amputation site on the left foot, there was a skin ulcer measuring $25 \times 20 \mathrm{~mm}$ with several red nodules, primarily on the anterior surface of the lower left leg (fig. 2). Skin biopsies were obtained from the red nodules on the lower leg and the skin ulcer on the left foot.

Histopathological examination of both lesion samples revealed tumor cells with eosinophilic cytoplasm and medium-to-large nuclei with distinct nucleoli extending through all layers of the dermis and into some fatty tissue. The tumor comprised an alveolar structure and had a solid proliferative area (fig. 3). Immunostaining was positive for vimentin, S-100, HMB-45, melanoma, and Melan-A. The MIB-1 index was 38.3\%. Based on these results, a diagnosis of recurrent malignant melanoma with in-transit metastasis was made.

\section{Treatment and Progress}

Subtotal integumentectomy was scheduled; however, 2 weeks later, the patient developed acute myocardial infarction and was hospitalized at Kariya Toyota General Hospital. Treatment for acute myocardial infarction continued for 1 month; thereafter, the patient was transferred to our department. As the myocardial infarction decreased the patient's tolerance to surgery, intravenous infusion of IFN- $\beta$ was decided as the treatment of choice. Initially, IFN- $\beta$ was administered at 3 million $U /$ day for 10 consecutive days. As a countermeasure against fever, which is a side effect of IFN- $\beta$, Loxonin ${ }^{\circledR}$ was orally administered three times per day on the days of infusion. Thereafter, the patient was treated as an outpatient, with intravenous infusion of IFN- $\beta 3$ million U/day once a week. Twenty-six weeks later, the skin ulcer on the fifth toe of the left foot was cured, the nodes on the left leg had disappeared, and only pigmentation was observed (fig. 4). After 1 year, the regimen was changed to intravenous infusion of IFN- $\beta 3$ million U/day once every 2 weeks. The patient's progress has been satisfactory, with no evidence of recurrence or metastasis at 1 year and 9 months after treatment (fig. 5). The patient continues to receive IFN- $\beta 3$ million U/day every 2 weeks. 
Arima et al.: A Case of Malignant Melanoma with In-Transit Metastasis That Responded to Intravenous Infusion of Interferon- $\beta$

\section{Discussion}

With regard to primary malignant melanomas of the skin, circulating tumor cells were reportedly detected in 32\% [1] of the patients with stage I disease using polymerase chain reaction analysis of melanocyte differentiation markers, and in $22 \%$ [2] of the patients with a tumor thickness of $<1 \mathrm{~mm}$ by screening according to cell size. These findings strongly suggest that malignant melanoma causes systemic dissemination of tumor cells in earlystage carcinogenesis. These cells may lie dormant for a long period in a state of growth arrest, but they eventually grow and become clinically detectable metastases [3]. Therefore, malignant melanoma is a systemic disease that causes dissemination of tumor cells during the early stage of carcinogenesis and it should be treated with adequate consideration of these biochemical characteristics.

Malignant melanoma is resistant to chemotherapy, and, at present, there is no effective chemotherapy. Therefore, a useful postoperative adjuvant therapy is much-needed. Reports from the USA and Europe deny the usefulness of postoperative adjuvant chemotherapy with dacarbazine and claim that the intravenous injection of IFN- $\alpha$ is the only treatment method with demonstrated supporting evidence [4]. In Japan, the most commonly used postoperative adjuvant therapy for patients with stage II/III malignant melanoma is DAVFeron therapy (combined chemotherapy with dacarbazine, nimustine, and vincristine) and local injection of IFN- $\beta$ into the primary lesion site. Additional regimes include IFN therapy with daily injections of IFN- $\beta$ and long-term therapy with IFN administered once every 2-4 weeks. However, there is no evidence supporting these treatments. The effectiveness of these postoperative adjuvant therapies was analyzed using data obtained from a national follow-up survey conducted by the Prognosis and Statistical Investigation Committee of the Japanese Skin Cancer Society. Long-term IFN therapy significantly improved survival in all cancer stages, whereas DAVFeron therapy and IFN therapy showed no effect $[5,6]$. However, some questions remain to be answered. First, is local injection of IFN- $\beta$ actually ineffective (or minimally effective) on cancer cells disseminated throughout the body? Second, following the injection of Patent Blue ${ }^{\circledR}$, which is used to obtain sentinel lymph node biopsies from near the site of scarring caused by surgical removal of the tumor, does the absence or lack of pigment flow indicate that IFN- $\beta$ has not been effectively administered? Moreover, it is believed that the anti-tumor action of IFN- $\beta$ involves direct inhibition of tumor cell growth via binding to tumor cells, with simultaneous indirect inhibition of tumor proliferation via activation of the host's immune system [7]. In our case, recurrent localized malignant melanoma on the fifth toe of the left foot with in-transit metastasis on the lower left leg responded to intravenous infusion of IFN- $\beta$. Therefore, intravenous infusion of IFN- $\beta$ may be more effective against malignant melanoma compared with local injection. We believe that the administration of IFN- $\beta$ should be reconsidered in Japan.

\section{References}

1 Mocellin S, Hoon D, Ambrosi A, Nitti D, Rossi CR: The prognostic value of circulating tumor cells in patients with melanoma: a systematic review and meta-analysis. Clin Cancer Res 2006;12:4605-4613.

2 De Giorgi V, Pinzani P, Salvianti F, et al: Application of a filtration and isolation-by-size technique for the detection of circulating tumor cells in cutaneous melanoma. J Invest Dermatol 2010;130:2440-2447.

3 Takata M: Does surgery cure melanoma? Re-evaluation of melanoma surgery based on a new progression model (in Japanese). Jpn J Dermatol 2012;122:2077-2084.

-4 Eggermont AM, Testori A, Marsden J, et al: Utility of adjuvant systemic therapy in melanoma. Ann Oncol 2009;20(suppl 6):30-34. 
Arima et al.: A Case of Malignant Melanoma with In-Transit Metastasis That Responded to Intravenous Infusion of Interferon- $\beta$

5 Fujisawa Y, Otsuka F: Japanese Melanoma Study Group: Does adjuvant therapy improve the prognosis of stage II and III melanoma patients? An analysis of 831 cases (in Japanese). Jpn J Dermatol 2012;122:23052311.

6 Fujisawa Y, Otsuka F, Yamamoto A, Yamazaki N, Saida T, Ishihara K: Statistics of melanoma in Japan: analysis of a 2006-2007 nationwide survey and a 2005-2010 follow-up survey (in Japanese). Skin Cancer (Japan) 2012;27:195-204.

7 Yamamoto A: Feron DAV combination chemotherapy (in Japanese). Skin Cancer (Japan) 1996;11:358-366.

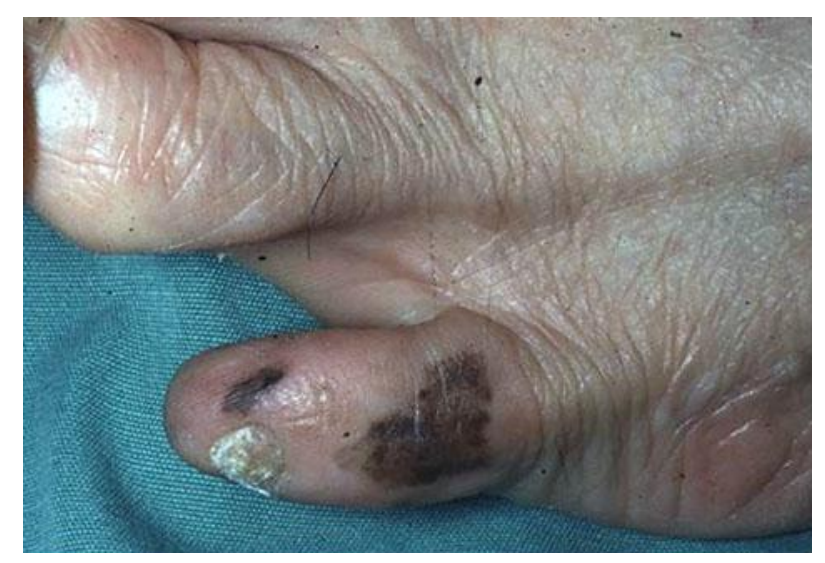

Fig. 1. Clinical feature from 14 years ago. A black mole on the fifth toe of the left foot. A diagnosis of malignant melanoma in situ was made.
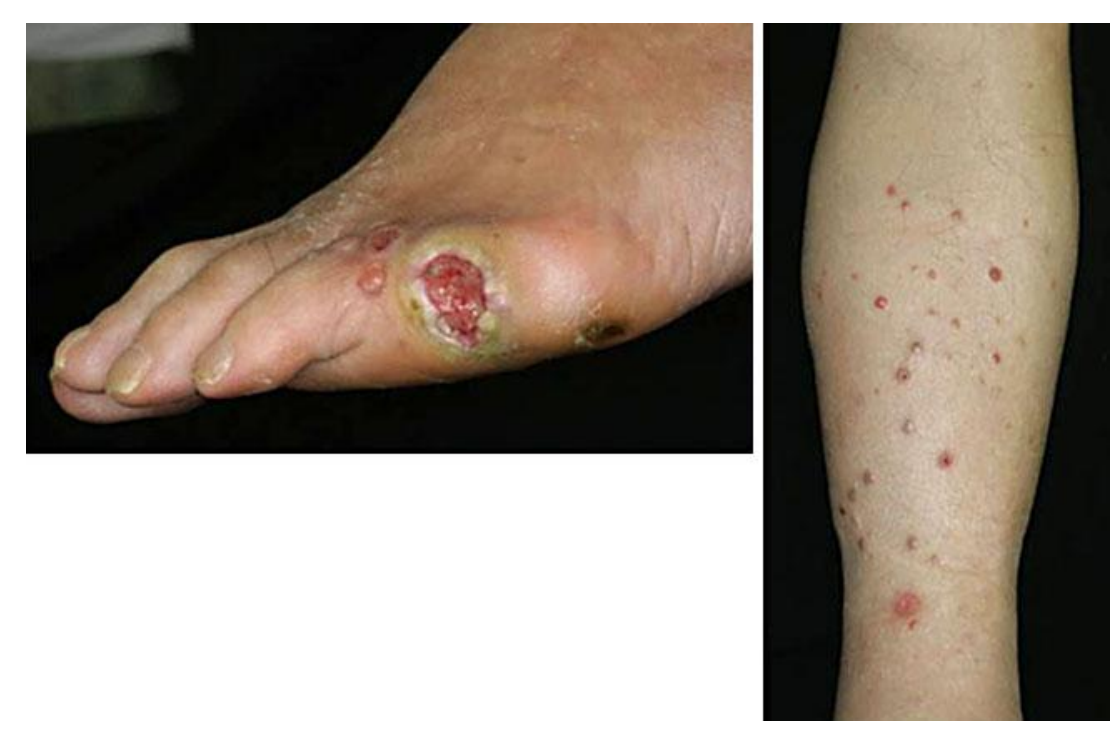

Fig. 2. Clinical feature at the time of re-examination. a A skin ulcer measuring $25 \times 20 \mathrm{~mm}$ is located on the amputated surface of the fifth toe of the left foot. $\mathbf{b}$ Multiple red nodules on the anterior surface of the lower left leg. 
Arima et al.: A Case of Malignant Melanoma with In-Transit Metastasis That Responded to Intravenous Infusion of Interferon- $\beta$
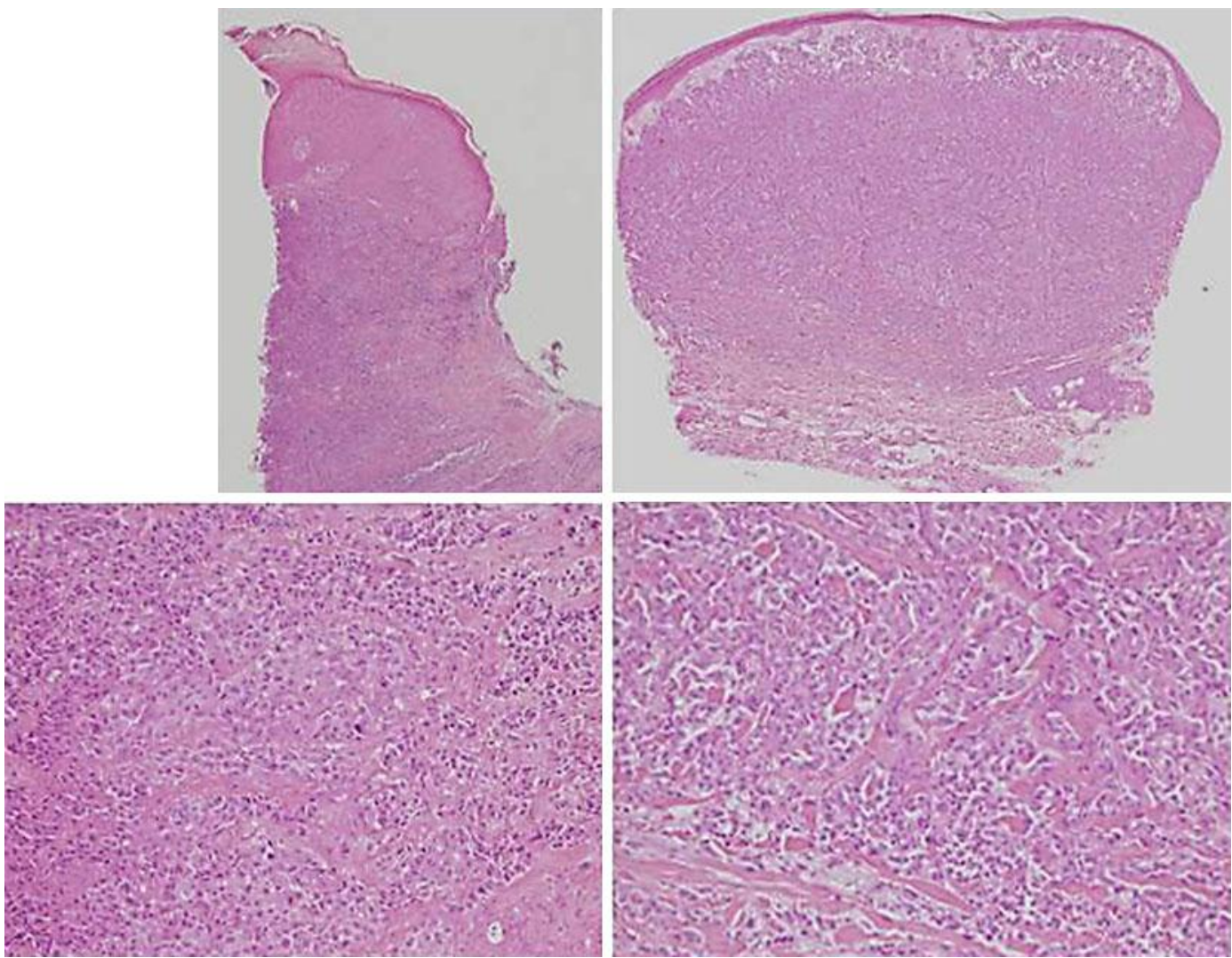

Fig. 3. Histopathology (HE stain). a Skin ulcer on the foot. b Nodules on the lower leg. Tumor cells with eosinophilic cytoplasm and medium-to-large nuclei with distinct nucleoli extend through all layers of the dermis and into some fatty tissue. The tumor comprises an alveolar structure and solid proliferative areas. 
Arima et al.: A Case of Malignant Melanoma with In-Transit Metastasis That Responded to Intravenous Infusion of Interferon- $\beta$
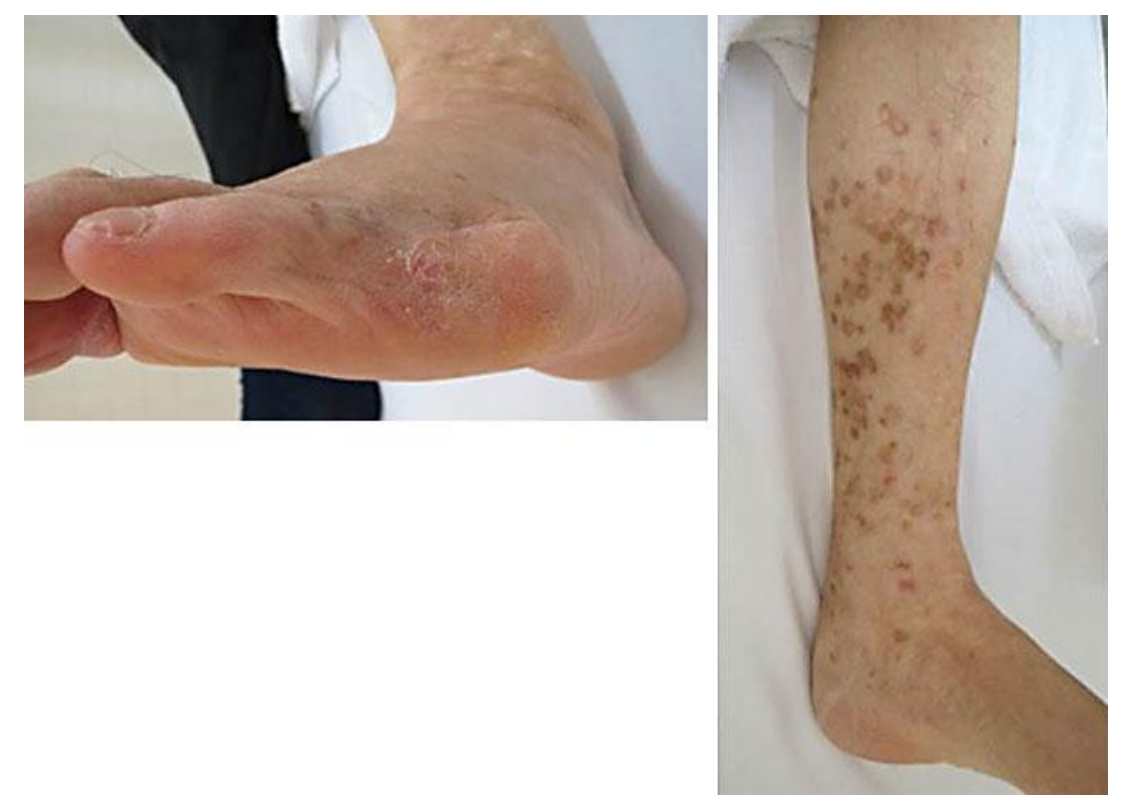

Fig. 4. Clinical feature after 26 weeks. a The skin ulcer on the amputated surface of the fifth toe of the left foot has healed. $\mathbf{b}$ The red nodules on the lower left leg have disappeared, and only pigmentation can be observed.
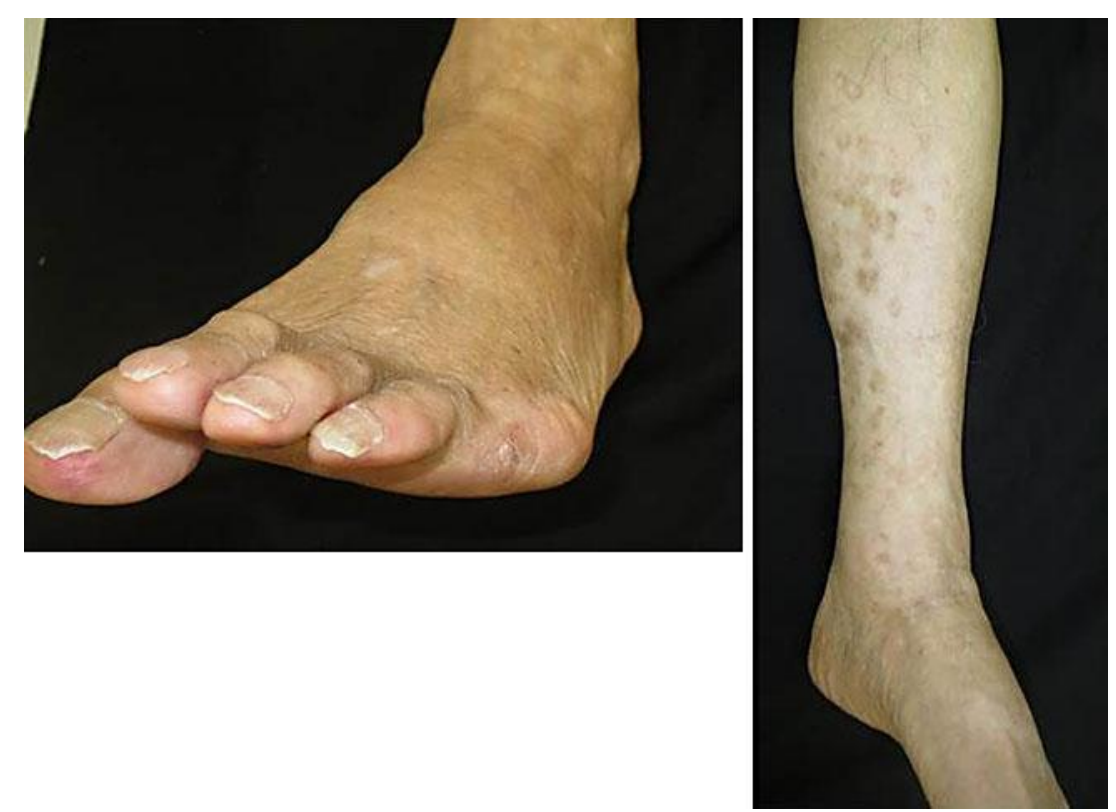

Fig. 5. Clinical feature at 1 year and 9 months. a There is no local recurrence on the fifth toe of the left foot. b The pigmentation on the lower left leg has lightened. 